\title{
ON A NONLINEAR ELLIPTIC BOUNDARY VALUE PROBLEM
}

\section{NGUYEN PHUONG CÁC}

ABSTRACT. Consider a bounded domain $G \subset R^{N}(N \geq 1)$ with smooth boundary $\Gamma$. Let $L$ be a uniformly elliptic linear differential operator. Let $\gamma$ and $\beta$ be two maximal monotone mappings in $R$. We prove that, when $\gamma$ satisfies a certain growth condition, given $f \in L^{2}(G)$ there is $u \in H^{2}(G)$ such that

$$
L u+\gamma(u) \ni f \text { a.e. on } G, \quad \text { and } \quad-\partial u / \partial \nu \in \beta\left(\left.u\right|_{\Gamma}\right) \text { a.e. on } \mathbf{r} \text {, }
$$

where $\partial u / \partial \nu$ is the conormal derivative associated with $L$.

1. Let $G \subset R^{N}(N \geq 1)$ be a bounded domain with smooth boundary $\Gamma$. Consider the uniformly elliptic linear operator

$$
\begin{aligned}
& L u=-D_{j}\left(a_{i j}(x) D_{i} u\right)+b_{i}(x) D_{i} u+c(x) u, \\
& a_{i j}=a_{j i} \in C^{1}(\bar{G}) ; b_{i}, c \in L^{\infty}(G) \quad(i, j=1,2, \cdots, N), \\
& a_{i j}(x) \xi_{i} \xi_{j} \geq c|\xi|^{2}, \quad c>0 \text { constant }, \forall x \in G, \xi \in R^{N} .
\end{aligned}
$$

(All functions and scalars that we consider are real.)

Let $\gamma: R \rightarrow 2^{R}$ be a maximal monotone mapping. The domain $D(\gamma)$ of $\gamma$ is the set of all numbers $s$ such that $\gamma(s) \neq \varnothing$. For each $s \in D(\gamma)$, $\gamma(s)$ is a closed interval and thus contains a unique element, which we denote by $\gamma^{0}(s)$, having smallest absolute value. We assume that the mapping $\gamma$ satisfies the condition

$$
\left|\gamma^{0}(s)\right| \geq \phi(s)|s|, \quad \forall s \in D(\gamma) \text { with } \lim _{|s| \rightarrow \infty} \phi(s)=\infty \text {. }
$$

It can be verified that $\gamma$ induces a maximal monotone mapping $\bar{\gamma}: L^{2}(G) \rightarrow$ $2^{L^{2}(G)}$ in a natural way:

$$
\bar{\gamma}(u)=\left\{v \in L^{2}(G) \mid v(x) \in \gamma(u(x)) \text { a.e. }\right\} \quad\left(u \in L^{2}(G)\right) .
$$

Received by the editors February 8, 1974 .

AMS (MOS) subject classifications (1970). Primary 35J20, 35J60, 35 J65;

Secondary 47H05, 47H10, 47H15.

Key words and phrases. Maximal monotone operators, Yosida approximation, nonlinear elliptic boundary value problem. 
Similarly, let $\beta: R \rightarrow 2^{R}$ be another maximal monotone mapping and let $\bar{\beta}$ be the mapping in $L^{2}(\Gamma)$ induced by $\beta$.

Proposition. Suppose that all the conditions on the operator $L$ and the mappings $\gamma$ and $\beta$ described above are satisfied. Suppose also that there exists $s_{0} \in D(\gamma) \cap D(\beta)$ with $0 \in \beta\left(s_{0}\right)$. Then for every given $f \in L^{2}(G)$, there exists $u \in H^{2}(G)$ such that

$$
\begin{aligned}
& L u+\bar{\gamma}(u) \ni f \text { in the sense of } L^{2}(G), \\
& -\partial u / \partial \nu=a_{i j} D_{i} u \cdot \cos \left(n, x_{j}\right) \in \bar{\beta}\left(u_{\left.\right|_{\Gamma}}\right),
\end{aligned}
$$

where $n$ is the outward normal to $\Gamma$.

Before proving this proposition in $\$ 2$, we would like to make a few comments. When (i) $\gamma \equiv 0$ and the bilinear form

$$
a(u, v)=\int_{G}\left(a_{i j} D_{i} u D_{j} v+b_{i} D_{i} u \cdot v+c u v\right) d x
$$

is coercive on $H^{1}(G)$, or (ii) $L u=-\Delta u+u$, the proposition has been proved by $H$. Brézis in [3] and [2] respectively. It seems to us that the general case, where no coercivity is assumed, cannot be immediately reduced to these cases.

The corresponding Dirichlet problem

$$
\begin{array}{r}
L u+\bar{\gamma}(u) \ni f, \\
u_{\Gamma}=0,
\end{array}
$$

has been studied by P. Hess [7], and condition (1) on $\gamma$ is similar to his condition in [7]. Our method can also be applied to this Dirichlet problem, and the argument will be simpler than the Neumann-type problem considered here. We feel that the main difference between our method as applied to the Dirichlet problem and that of $P$. Hess lies in proving the existence of a solution for the approximate equation. He uses a theorem on the solvability of a functional equation involving a demicontinuous mapping of type $\left(S^{+}\right)$ which is similar to one established by him earlier [6, Theorem 1], using a homotopy argument. Instead, we shall use the well-known Schauder fixed point theorem (see e.g. [4, p. 105]).

2. The proof makes use of the concept of the Yosida approximation. Let $U: H \rightarrow 2^{H}$ be a maximal monotone operator in a Hilbert space $H$. Then for every $\epsilon>0,(I+\epsilon U)^{-1}$ is a nonexpansive mapping defined on all of $H$. The mapping

$$
U_{\epsilon}=\left[I-(I+\epsilon U)^{-1}\right] / \epsilon
$$


is called the Yosida approximation of $U$ (at $\epsilon$ ). $U_{\epsilon}$ is Lipschitzian with Lipschitz constant $2 / \epsilon$ and monotone. For more details on Yosida approximations we refer the reader to $M$. Crandall and A. Pazy [5], or T. Kato [8]. In the case of the maximal monotone mappings $\gamma$ and $\beta$ introduced in $\$ 1$, it can be verified that the Yosida approximation of $\bar{\gamma}$ for example is generated by $\gamma_{\epsilon}$.

Proof of the proposition. We observe that by shifting and changing variable, we can assume without loss of generality that $0 \in \gamma(0)$ and $0 \in \beta(0)$. Then $\gamma_{\epsilon}(0)=\beta_{\epsilon}(0)=0$. The proof consists of proving that the approximate problem

$$
\begin{aligned}
L u+\dot{\bar{\gamma}}_{\epsilon}(u) & =f, \\
-\partial u / \partial \nu & =\bar{\beta}_{\epsilon}\left(u_{\left.\right|_{\Gamma}}\right)
\end{aligned}
$$

has a solution $u_{\epsilon} \in H^{2}(G)$ for all $\epsilon>0$ sufficiently small. We then pass to the limit as $\epsilon \downarrow 0$ using estimates for $u_{\epsilon}$ independent of $\epsilon$.

I. Proof that the approximate problem has a solution. Let

$$
\begin{aligned}
L^{\prime} u & =-D_{j}\left(a_{i j} D_{i} u\right), \quad L^{\prime \prime}=L-L^{\prime}, \\
a^{\prime}(u, v) & =\int_{G} a_{i j} D_{i} u D_{j} v d x \quad\left(u, v \in H^{1}(G)\right) .
\end{aligned}
$$

For $u$ given in $H^{1}(G)$, the linear form

$$
w \rightarrow a^{\prime}(u, w)+\int_{G}\left[\gamma_{\epsilon}(u)+u\right] w d x+\int_{\Gamma} \beta_{\epsilon}\left(u_{\mid \Gamma}\right) w d \Gamma \quad\left(w \in H^{1}(G)\right),
$$

is continuous on $H^{1}(G)$, so that there is an element $\mathbb{P} u \in\left[H^{1}(G)\right]^{\prime}$ with

$$
\left\langle\mathbb{Q}_{u}, w\right\rangle=a^{\prime}(u, w)+\int_{G}\left[\gamma_{\epsilon}(u)+u\right] w d x+\int_{\Gamma} \beta_{\epsilon}\left(u_{\left.\right|_{\Gamma}}\right) w d \Gamma \quad\left(w \in H^{1}(G)\right) .
$$

It can be verified that the mapping $u \rightarrow \mathbb{Q} u$ is bounded, hemicontinuous, strictly monotone and coercive. Therefore (see [4, Theorem 1] or [9, Chapter 2, Theorem 2.1]) for every given $v \in H^{1}(G)$ there exists a unique $u \in H^{1}(G)$ such that for all $w \in H^{1}(G)$

$$
\begin{aligned}
\left\langle\mathfrak{Q}_{u}, w\right\rangle & =a^{\prime}(u, w)+\int_{G}\left[\gamma_{\epsilon}(u)+u\right] w d x+\int_{\Gamma} \beta_{\epsilon}\left(u_{\mid \Gamma}\right) w d \Gamma \\
& =\int_{G}\left(f-L^{\prime \prime} v+v\right) w d x .
\end{aligned}
$$

We then deduce that the boundary value problem

$$
\begin{gathered}
L^{\prime} u+\gamma_{\epsilon}(u)+u=f-L^{\prime \prime} v+v \text { in the sense of } \mathcal{D}^{\prime}(G), \\
-\partial u / \partial \nu=\beta_{\epsilon}\left(u_{\left.\right|_{\Gamma}}\right)
\end{gathered}
$$

has a unique solution $u_{v} \in H^{1}(G)$. If we bring $\gamma_{\epsilon}(u)$ to the right-hand side in equation (9), then it follows from [3, Theorem I. 10], that $u_{v} \in H^{2}(G)$. 
It now suffices to show that the mapping $T_{\epsilon}: v \rightarrow u_{v}$ in $H^{1}(G)$ has a fixed point.

(a) The mapping $T_{\epsilon}$ is continuous. I.et $v_{1}, v_{2} \in H^{1}(G)$ and $u_{1}=T_{\epsilon}\left(v_{1}\right)$, $u_{2}=T_{\epsilon}\left(v_{2}\right)$. The continuity of $T_{\epsilon}$ can be seen by taking the difference of equations (9) corresponding to $v_{1}$ and $v_{2}$ and then taking the inner product in $L^{2}(G)$ of this with $u_{1}-u_{2}$.

(b) There is an integer $K>0$ and an $\epsilon_{0}>0$ such that $T_{\epsilon}\left(0<\epsilon<\epsilon_{0}\right)$ maps the closed ball $B_{K}(0)$ of $H^{1}(G)$ into itself. If not, for each $n=1,2, \cdots$ there are $\epsilon_{n}$ with $0<\epsilon_{n}<n^{-1}, v_{n}$ with $\left\|v_{n}\right\|_{1} \leq n, u_{n}$ with $\left\|u_{n}\right\|_{1}>n$ (where $\|\cdot\|_{1}$ is the norm in $H^{1}(G)$ ) satisfying (9) and (10) with $\epsilon=\epsilon_{n}$. Taking the inner product in $L^{2}(G)$ of (9) with $n^{-1} w_{n}=n^{-2} u_{n}$ we obtain

$$
\begin{gathered}
a^{\prime}\left(w_{n}, w_{n}\right)+\int_{\Gamma} n^{-2} \beta_{\epsilon_{n}}\left(u_{n}\right) u_{n} d \Gamma+\int_{G} n^{-2} \gamma_{\epsilon_{n}}\left(u_{n}\right) u_{n} d x+\left\|w_{n}\right\|_{0}^{2} \\
=\left\|n^{-1}\left(f-L^{\prime \prime} v_{n}+v_{n}\right)\right\|_{0} \cdot\left\|w_{n}\right\|_{0},
\end{gathered}
$$

where $\|\cdot\|_{0}$ is the norm in $L^{2}(G)$. From this we deduce

$$
\begin{gathered}
1<\left\|w_{n}\right\|_{1}<C \quad(n=1,2, \ldots), \\
\int_{G} n^{-2} \gamma_{\epsilon_{n}}\left(u_{n}\right) u_{n} d x<C \quad(n=1,2, \ldots) .
\end{gathered}
$$

Here and in the sequel $C$ denotes various positive constants independent of $\gamma, \beta, \epsilon$. Now taking the inner product in $L^{2}(G)$ of (9) with $n^{-2} \gamma_{\epsilon_{n}}\left(u_{n}\right)$ we obtain (for justification see [1] or [10, Appendix I] for a special case), recalling $w_{n}=n^{-1} u_{n}$ :

$$
\begin{gathered}
\int_{G} \gamma_{\epsilon_{n}^{\prime}}^{\prime}\left(u_{n}\right) a_{i j} D_{i} w_{n} D_{j} w_{n} d x+\int_{\Gamma} n^{-2} \beta_{\epsilon_{n}}\left(u_{n}\right) \gamma_{\epsilon_{n}}\left(u_{n}\right) d \Gamma+\int_{G} n^{-2} \gamma_{\epsilon_{n}}\left(u_{n}\right) u_{n} d x \\
+\left\|n^{-1} \gamma_{\epsilon_{n}}\left(u_{n}\right)\right\|_{0}^{2} \leq\left\|n^{-1}\left(f-L^{\prime \prime} v_{n}+v_{n}\right)\right\|_{0} \cdot\left\|n^{-1} \gamma_{\epsilon_{n}}\left(u_{n}\right)\right\|_{0} .
\end{gathered}
$$

The third integral is nonnegative. Since $0 \in \gamma(0), \gamma_{\epsilon_{n}}(\cdot)$ is monotone increasing so that $\gamma_{\epsilon_{n}}^{\prime}\left(u_{n}(x)\right) \geq 0$ a.e. and the first integral is also nonnegative. Moreover, we observe that if $u_{n}(x)=0$ then $\beta_{\epsilon_{n}}\left(u_{n}\right) \gamma_{\epsilon_{n}}\left(u_{n}\right)=0$, and if $u_{n}(x) \neq 0$ then

$$
\beta_{\epsilon_{n}}\left(u_{n}\right) \gamma_{\epsilon_{n}}\left(u_{n}\right)=\beta_{\epsilon_{n}}\left(u_{n}\right) u_{n} \cdot \gamma_{\epsilon_{n}}\left(u_{n}\right) u_{n} \cdot u_{n}^{-2} \geq 0
$$

so that the second integral is also nonnegative. We then deduce that

$$
\left\|n^{-1} \gamma_{\epsilon_{n}}\left(u_{n}\right)\right\|_{0}<C \quad(n=1,2, \ldots) .
$$

We now write 


$$
\begin{aligned}
& L^{\prime} w_{n}+w_{n}=n^{-1}\left(f-L^{\prime \prime} v_{n}+v_{n}-\gamma_{\epsilon_{n}}\left(u_{n}\right)\right), \\
& -\partial w_{n} / \partial \nu=n^{-1} \beta_{\epsilon_{n}}\left(u_{n \mid \Gamma}\right) .
\end{aligned}
$$

Since the right-hand side of (13) remains bounded in $L^{2}(G)$, it follows from [3, Theorem I.10], that $\left\|w_{n}\right\|_{2}<C(n=1,2, \ldots)$, where $\|\cdot\|_{2}$ denotes the norm in $H^{2}(G)$. Because the imbedding of $H^{2}(G)$ into $H^{1}(G)$ is compact, we can extract a subsequence, still denoted by $\left\{w_{n}\right\}$, of $\left\{w_{n}\right\}$ such that $w_{n}$ converges strongly in $H^{1}(G)$ to $w$ and $w_{n}$ converges a.e. on $G$ to $w$. Since $\|w\|_{1} \geq 1(n=1,2, \cdots), w(x) \neq 0$ on a subset of $G$ of nonzero measure. We shall see that this contradicts condition (1) on $\gamma$ and (12). In fact, putting $s_{n}(x)=\left(I+\epsilon_{n} \gamma\right)^{-1} u_{n}(x)$, we obtain with $t_{n}(x) \epsilon \gamma\left(s_{n}(x)\right)$

$$
\begin{aligned}
u_{n}(x) & =s_{n}(x)+\epsilon_{n} t_{n}(x), \\
n^{-2} \gamma_{\epsilon_{n}}\left(u_{n}(x)\right) u_{n}(x) & =n^{-2} u_{n}(x) t_{n}(x)=\left|w_{n}(x)\right| \cdot n^{-1}\left|t_{n}(x)\right| .
\end{aligned}
$$

Consider $x \in G$ with $\lim _{n}\left|w_{n}(x)\right|>0$, i.e. $\lim _{n}\left|u_{n}(x)\right|=\infty$. Then

$$
\lim _{n} n^{-1}\left|t_{n}(x)\right|=\infty \text {. }
$$

For otherwise there would be a subsequence such that

$$
\sup _{k} n_{k}^{-1}\left|t_{n_{k}}(x)\right|<\infty .
$$

From (15) it then follows that

$$
\lim _{k} \inf n_{k}^{-1}\left|s_{n_{k}}(x)\right| \geq \lim _{k} n_{k}^{-1}\left|u_{n_{k}}(x)\right|>0 .
$$

By condition (1) on $\gamma$,

$$
n_{k}^{-1}\left|t_{n_{k}}(x)\right| \geq n_{k}^{-1}\left|\gamma^{0}\left(s_{n_{k}}(x)\right)\right|>\phi\left(s_{n_{k}}(x)\right) n_{k}^{-1}\left|s_{n_{k}}(x)\right| \text {. }
$$

Since $\lim _{k}\left|s_{n_{k}}(x)\right|=\infty, \lim _{k} \phi\left(s_{n_{k}}(x)\right)=\infty$. This together with (17) shows that

$$
\lim _{k} n_{k}^{-1}\left|t_{n_{k}}(x)\right|=\infty
$$

and we thus arrive at a contradiction. From (16) we therefore see that

$$
\lim _{n} n^{-2} \gamma_{\epsilon_{n}}\left(u_{n}(x)\right) u_{n}(x)=\infty
$$

on a subset of $G$ of nonzero measure. By Fatou's lemma, this contradicts (12).

(c) The mapping $T_{\epsilon}\left(0<\epsilon<\epsilon_{0}\right)$ of $B_{K}(0)$ into itself is relatively compact. In fact, by an argument similar to that in the last step, we see 
that for all $v \in B_{K}(0),\left\|T_{\epsilon}(v)\right\|_{2}<C$. Since the imbedding of $H^{2}(G)$ into $H^{1}(G)$ is compact, we deduce that the closure of $T_{\epsilon}\left(B_{K}(0)\right)$ is compact.

Thus by the Schauder fixed point theorem [4,p. 105], $T_{\epsilon}\left(0<\epsilon<\epsilon_{0}\right)$ has a fixed point in $B_{K}(0)$.

II. Passing to the limit as $\epsilon \downarrow \mathbb{0}$. Using the same argument as in Step I (b) above (take the inner product in $L^{2}(G)$ of (7) with $u$ and then with $\bar{\gamma}_{\epsilon}(u)$ ), we see that there is a constant $C$ independent of $\epsilon$ such that a solution $u_{\epsilon}$ of (7) and (8) satisfies

$$
\left\|u_{\epsilon}\right\|_{2}<C, \quad\left\|\bar{\gamma}_{\epsilon}\left(u_{\epsilon}\right)\right\|_{0}<C \quad\left(0<\epsilon<\epsilon_{0}\right) .
$$

Since the mapping $\left.u \rightarrow u\right|_{\Gamma}$ of $H^{1}(G)$ onto $H^{1 / 2}(\Gamma) \subset L^{2}(\Gamma)$ is continuous, we can extract a subsequence $\left\{u_{\epsilon_{n}}\right\}$ with the following properties

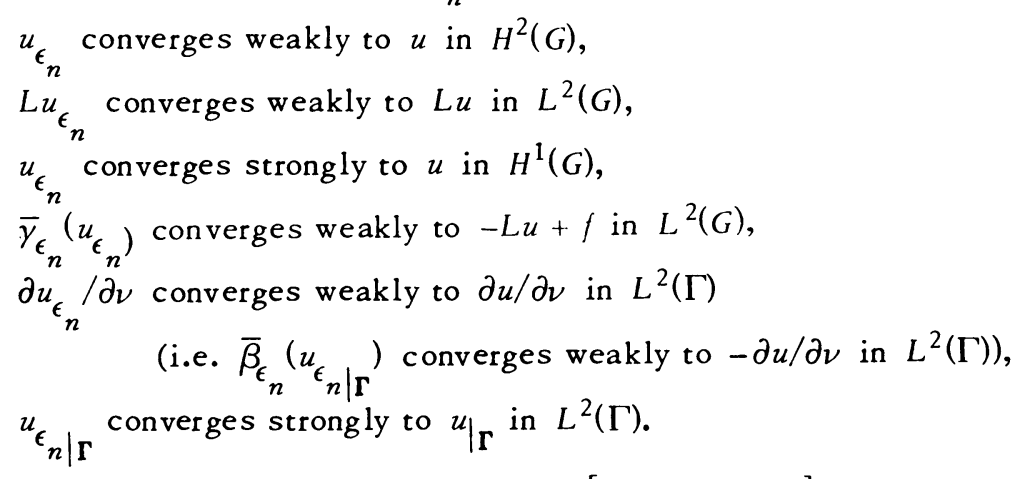

From a property of Yosida approximations [8, Lemma 4.5], it then follows that

$$
u \in D(\bar{y}), \quad-L u+f \in \bar{\gamma}(u) ; \quad u_{\mathbf{\Gamma}_{\mathbf{\Gamma}}} \in D(\bar{\beta}), \quad-\partial u / \partial \nu \in \bar{\beta}\left(u_{\mid \mathbf{r}}\right)
$$

and the proof is complete.

From the proposition we deduce the following

Corollary. Suppose that the conditions in the proposition are satisfied. Then for any $k_{1} \geq 0, k_{2}>0$, the boundary value problem

$$
L u+\bar{\gamma}(u) \ni f, \quad-k_{1} u-k_{2} \partial u / \partial \nu \in \bar{\beta}\left(u_{\left.\right|_{\Gamma}}\right)
$$

has a solution $u \in H^{2}(G)$.

Proof. The boundary condition can be written as

$$
-\partial u / \partial \nu \in k_{1} k_{2}^{-1} u+k_{2}^{-1} \beta\left(u_{\mid \Gamma}\right)
$$

On the other hand, it can be verified that $k_{1} k_{2}^{-1} I+k_{2}^{-1} \beta$ is a maximal 
monotone mapping in $R$, using the well-known fact that a monotone mapping $U$ in a Hilbert space $H$ is maximal if and only if for all $\lambda>0$ the range of $I+\lambda U$ is the whole of $H$ (see e.g. [2]).

\section{REFERENCES}

1. H. Brézis, Nouveaux théorèmes de régularité pour les problèmes unilatéraux, Publication R. C. P. No 25, Strasbourg, 1971.

2. - Monotonicity methods in Hilbert spaces and some applications to nonlinear partial differential equations, Contribution to Nonlinear Functional Analysis, Academic Press, New York, 1971, pp. 101-156.

3. - Problemes unilatéraux, J. Math. Pures. Appl. 51 (1972), 1-168.

4. F. E. Browder, Problèmes non linéaires, Séminaire de Mathématiques Supérieures, no. 15, (Été, 1965), Les Presses de l'Université de Montréal, Montréal, Québec, 1966. MR $40 \# 3380$.

5. M. Crandall and A. Pazy, Semigroups of nonlinear contractions and dissipative sets, J. Functional Analysis 3 (1969), 376-418. MR 39 \#4705.

6. P. Hess, On nonlinear mappings of monotone type homotopic to odd operators, J. Functional Analysis 11 (1972), 138-167.

7. - On a unilateral problem associated with elliptic operators, Proc. Amer. Math. Soc. 39 (1973), 94-100.

8. T. Kato, Accretive operators and nonlinear evolution equations in Banach spaces, Proc. Sympos. Pure Math., vol. 18, part 1, Amer. Math. Soc., Providence, R.I., 1970, pp. 138-161. MR $42 \# 6663$.

9. J.-L. Lions, Quelques méthodes de résolution des problèmes aux limites non linéaires, Dunod; Gauthier-Villars, Paris, 1969. MR 41 \#4326.

10. G. Stampacchia, Équations elliptiques du second ordre à coefficients dis. continus, Séminaire de Mathématiques Supérieures, no. 16 (Été, 1965), Les Presses de l'Université de Montréal, Montréal, Québec, 1966. MR $40 \# 4603$. 